INRA Prod. Anim., $1995,8(3), 189-195$
M. LESSIRE, N. REVOL*, F. RUDEAUX ${ }^{* * *}$, J.M. HALLOUIS

INRA Station de Recherches Avicoles 37380 Nouzilly

* Ecole Supérieure d'Agriculture 55 rue Rabelais 49000 Angers

**: SANDERS Aliments 17 quai de l'Industrie 91200 Athis-Mons

\section{Valeur énergétique des aliments chez la poule pondeuse}

\section{Plus de deux millions de tonnes d'aliments sont fabriquées en France pour nourrir les poules pondeuses en retenant les caractéristiques nutritionnelles mesurées sur coq adulte. Or le coq n'est pas toujours la bonne référence de formulation et la valeur énergétique des aliments peut varier en fonction du stade de production de la poule.}

Le résultat technico-économique d'un élevage avicole dépend, pour une large part, du niveau énergétique ou énergie métabolisable (EM) de l'aliment défini par le formulateur (Pesti 1994). De plus, une décomposition rapide du coût alimentaire (60 à $70 \%$ du coût de la production) en énergie et protéines (Leclercq 1985) montre que ces composantes représentent respectivement 62 et $38 \%$ du coût d'un aliment moyen renfermant $3000 \mathrm{kcal}$ d'EM et $200 \mathrm{~g}$ de protéines par $\mathrm{kg}$. En outre, les poulets et les poules ajustant leur quantités ingérées sur la concentration énergétique de la ration, les apports en nutriments (acides aminés, minéraux...) s'expri-

\section{Résumé}

Le coq adulte est le modèle expérimental utilisé par le nutritionniste pour déterminer la valeur énergétique des aliments chez les volailles. Ces données sont retenues par les industriels pour formuler les rations destinées aux poulets, poules, dindonneaux... Or, il est démontré que le coq adulte ne reflète pas toujours parfaitement l'utilisation digestive des aliments chez le poulet et les autres espèces aviaires.

L'étude bibliographique que nous présentons montre qu'il peut en être de même chez la femelle, même si l'interprétation des résultats est délicate compte tenu des diverses méthodologies rencontrées.

Dans une première série d'essais, nous définissons une méthode de bilan digestif utilisable chez la poule. L'optimum, en terme de précision, de répétabilité et de facilité de réalisation, comprend 3 jours d'accoutumance à l'aliment, $17 \mathrm{~h}$ de jeûne, 7 jours d'alimentation et $17 \mathrm{~h}$ de jeûne. Dans une deuxième série d'essais, nous montrons que la valeur énergétique d'un aliment complet est peu modifiée $( \pm 2,5 \%)$ lorsque l'âge des oiseaux varie de 16 semaines à 70 semaines pour des productions journalières d'œuf comprises entre 0 et $60 \mathrm{~g}$.

De même, les valeurs observées chez le coq sont très proches de celles obtenues chez la femelle. Ces faibles variations ont cependant une importance non négligeable pour l'industriel, et il conviendrait d'en vérifier l'ampleur sur d'autres aliments moins conventionnels. ment pour une teneur en EM donnée de la ration. Il convient donc d'estimer parfaitement ce paramètre et, pour cela, de nombreuses méthodes in vivo (Sibbald 1976a, Farell 1978, Kussaibati et Leclercq 1985, Bourdillon et al 1990a, b, Lessire, 1990) et in vitro : équations de prédiction (Carré et Rozo 1990) ont été proposées tant pour les aliments complets que pour les matières premières constitutives des rations. Ces méthodes permettent de caractériser précisément la plupart des matières premières $\mathrm{y}$ compris celles dont l'utilisation est récente: graines oléagineuses (Lessire et al 1988, Leclercq et al 1989) ou d'en préciser les facteurs de variation (Conan et al 1992).

La critique que l'on peut cependant formuler à l'encontre de ces travaux est qu'ils retiennent pour seul modèle animal le coq adulte ; or celui-ci ne consomme qu'une proportion marginale des 7,7 millions de tonnes d'aliments composés produits en France qui sont en fait pratiquement exclusivement destinés à des poules pondeuses et à des oiseaux en croissance: poulets et dindonneaux. En outre, il a été montré à maintes reprises que le coq n'est pas le meilleur estimateur de la valeur nutritionnelle des aliments destinés aux volailles, qu'il s'agisse de tourteaux ou de céréales (Leclercq 1979) ou des matières grasses (voir revue de Wiseman 1984). Il faut donc s'interroger sur la validité des valeurs obtenues sur coqs pour estimer l'énergie métabolisable des aliments et matières premières destinés à la femelle en ponte.

Il nous a donc paru intéressant d'analyser les valeurs énergétiques des aliments publiées pour la poule pondeuse et surtout de les comparer à celles observées chez le coq adulte et chez le poulet de chair. Dans un deuxième temps, nous développons une 
méthode de mesure de l'énergie métabolisable des aliments spécifique à la poule, puis nous présentons l'incidence de certains facteurs de variation tels que l'âge et le sexe de l'animal.

\section{1 / Etude bibliographique}

Le coq adulte étant la référence de nombreux auteurs pour la détermination de l'EM des aliments destinés aux volailles, notre premier objectif a été de recenser les valeurs observées simultanément chez cet animal et chez la poule pondeuse. Les données obtenues ainsi que leur origine sont présentées au tableau 1, sans mentionner le système énergétique retenu (EM apparente ou vraie, corrigée ou non pour un bilan azoté nul), les méthodologies mises en œuvre ou la composition des matières premières. Il en résulte que les valeurs présentées pour un même aliment sont difficilement comparables d'un auteur à l'autre.

La valeur EM des céréales : mais, blé, avoine ne varie pas en fonction de l'animal considéré : coq ou poule pondeuse White Leghorn (Sibbald 1976b) alors que les issues de blé, riches en fibres, semblent mieux utilisées par la poule que par le coq, même si les différences observées : 137 et $110 \mathrm{kcal}$ ne sont pas significatives.

Pour le tourteau de soja, deux auteurs mentionnent des valeurs indépendantes du sexe de l'oiseau : Sibbald (1976b), Parson et al (1982), alors que pour Askbrant (1988) ce tourteau a, chez la poule, une valeur inférieure à celle mesurée chez le coq : 2502 vs $2734 \mathrm{kcal} / \mathrm{kg}$. Il apparaît donc difficile de conclure à une éventuelle différence d'utilisation digestive du tourteau de soja chez le coq et la poule. A l'inverse, pour les tourteaux de colza et le pois, les valeurs sont statistiquement identiques, même si des différences importantes sont mentionnées. Les valeurs de la farine de poisson et de deux aliments complets sont également identiques chez les deux oiseaux. En définitive, la poule pondeuse semble donc utiliser les aliments comme le coq.

Cependant, d'un point de vue méthodologique, on peut s'interroger quant à la validité des valeurs et comparaisons présentées. En effet, Askbrant (1988) utilise des coqs gavés et des poules nourries ad libitum. Pour les autres auteurs la méthode de bilan digestif utilisée repose sur un gavage unique d'une très faible quantité d'aliment (environ 1/3 de l'ingéré spontané quotidien), les oiseaux restant ensuite à jeun pendant 48 à $72 \mathrm{~h}$. Ce protocole expérimental (Sibbald 1976a) conduit, chez le coq, à des résultats similaires à ceux obtenus avec des schémas d'alimentation ad libitum (Lessire 1990), ce qui n'a pas été démontré avec la poule pondeuse dont la production d'œufs devrait diminuer avec les faibles niveaux d'ingestion pratiqués. La quantité d'œuf produite n'est d'ailleurs jamais indiquée par les auteurs. Il nous est donc paru opportun de mentionner dans le tableau 2 les études comparatives effectuées entre poule et poulet. En effet ces comparaisons plus nombreuses ont été réalisées en utilisant des techniques de bilan avec alimentation ad libitum des animaux, et des régimes la plupart du temps équilibrés.

D’une façon générale, la poule utilise les céréales mieux que le poulet. Les écarts entre les deux oiseaux peuvent atteindre $700 \mathrm{kcal}$ dans le cas de l'orge (Petersen et al 1976), cette matière première est en effet riche en $\beta$ glucanes, polysaccharides non amylacés solubles, capables de réduire la digestibilité

Tableau 1. Valeur énergétique (kcal/kg) des aliments chez la poule pondeuse et le coq adulte.

\begin{tabular}{|l|c|c|c|c|}
\hline & Poule & Coq & Signification & Référence \\
\hline Céréales et Sous produits & & & & \\
Maiss & $4180 \mathrm{G} *$ & $4187 \mathrm{G}$ & $\mathrm{ns}$ & Sibbald 1976b \\
Blé & $3910 \mathrm{G}$ & $3920 \mathrm{G}$ & $\mathrm{ns}$ & Sibbald 1976b \\
Avoine & $3590 \mathrm{G}$ & $3580 \mathrm{G}$ & $\mathrm{ns}$ & Sibbald 1976b \\
Son de blé & $1956 \mathrm{G}$ & $1819 \mathrm{G}$ & $\mathrm{ns}$ & Sibbald 1976b \\
Remoulages de blé & $2977 \mathrm{G}$ & $2867 \mathrm{G}$ & $\mathrm{ns}$ & Sibbald 1976b \\
Matières premières & & & & \\
riches en protéines & & & & \\
Tourteau de soja & $3243 \mathrm{G}$ & $3228 \mathrm{G}$ & $\mathrm{ns}$ & Sibbald 1976b \\
& $2714 \mathrm{AL}$ & $2683 \mathrm{AL}$ & $\mathrm{ns}$ & Parson et al 1982 \\
T. colza 0 hiver & $2502 \mathrm{AL}$ & $2734 \mathrm{G}$ & $\mathrm{s}$ & Askbrant 1988 \\
T. colza printemps & $1660 \mathrm{AL}$ & $1724 \mathrm{G}$ & $\mathrm{ns}$ & Askbrant 1988 \\
T. colza 0 0 & $1830 \mathrm{AL}$ & $1758 \mathrm{G}$ & $\mathrm{ns}$ & Askbrant 1988 \\
Pois & $2080 \mathrm{AL}$ & $1928 \mathrm{G}$ & $\mathrm{ns}$ & Askbrant 1988 \\
Farine de poisson & $2708 \mathrm{AL}$ & $2921 \mathrm{G}$ & $\mathrm{ns}$ & Askbrant 1988 \\
Aliments complets & $3084 \mathrm{G}$ & $3030 \mathrm{G}$ & $\mathrm{ns}$ & Sibbald 1976b \\
& $3368 \mathrm{G}$ & $3398 \mathrm{G}$ & $\mathrm{ns}$ & Sibbald $1976 \mathrm{~b}$ \\
\hline
\end{tabular}

$* \mathrm{G}=$ animaux gavés, $\mathrm{AL}=$ animaux nourris ad libitum 
Tableau 2. Valeur énergétique $(\mathrm{kcal} / \mathrm{kg})$ des aliments chez la poule pondeuse et le poulet (animaux nourris ad libitum).

\begin{tabular}{|c|c|c|c|c|}
\hline & Poule & Poulet & Signification & Référence \\
\hline \multicolumn{5}{|l|}{ Céréales et sous produits } \\
\hline \multirow[t]{3}{*}{ Mais } & 3310 & 3480 & & Hoshii et al $1970^{(1)}$ \\
\hline & 3490 & 3110 & $\mathrm{~s}$ & Petersen et al $1976^{(2)}$ \\
\hline & 3584 & 3284 & $\mathrm{~s}$ & Charalambous et Daghir $1976^{(3)}$ \\
\hline \multirow[t]{2}{*}{ Blé } & 3140 & 3060 & $\mathrm{~ns}$ & Petersen et al $1976^{(2)}$ \\
\hline & 3470 & 3315 & ns & Daghir et Nathanael $1974^{(4)}$ \\
\hline \multirow[t]{2}{*}{ Orge } & 3050 & 2350 & $\mathrm{~s}$ & Petersen et al $1976^{(2)}$ \\
\hline & 2980 & 2860 & $\mathrm{~ns}$ & Daghir et Nathanael $1974^{(4)}$ \\
\hline Triticale & 3253 & 3153 & ns & Daghir et Nathanael $1974^{(4)}$ \\
\hline \multirow[t]{2}{*}{ Son de blé } & 2270 & 2250 & & Hoshii et al $1970^{(1)}$ \\
\hline & 1180 & 1440 & $\mathrm{~s}$ & Petersen et al $1976^{(2)}$ \\
\hline Son de riz & 1690 & 1720 & & Hoshii et al $1970^{(1)}$ \\
\hline DDGS maïs & 2150 & 1650 & $\mathrm{~s}$ & Lilburn et Jensen $1984^{(1)}$ \\
\hline Coques d'orge & 1220 & 530 & $\mathrm{~s}$ & Petersen et al $1976^{21}$ \\
\hline \multicolumn{5}{|l|}{$\begin{array}{l}\text { Matières premières } \\
\text { riches en protéines }\end{array}$} \\
\hline \multirow[t]{3}{*}{ T. de soja } & 2300 & 2280 & & Hoshii et al $1970^{11}$ \\
\hline & 2640 & 1650 & $\mathrm{~s}$ & Petersen et al $1976^{(2)}$ \\
\hline & 2522 & 2513 & ns & Horani et Daghir $1975^{(5)}$ \\
\hline T. de colza & 1782 & 1313 & s & Lodhi et al $1969^{(6)}$ \\
\hline T. d'arachide & 2533 & 1984 & s & Charalambous et Daghir $1976^{(3)}$ \\
\hline T. de sésame & 2747 & 2690 & $\mathrm{~ns}$ & Horani et Daghir $1975^{(5)}$ \\
\hline T. de carthame & 2130 & 1250 & s & Petersen et al $1976^{2}$ \\
\hline Pois & 2850 & 2140 & $\mathrm{~s}$ & Petersen et al $1976^{2 i}$ \\
\hline \multirow[t]{2}{*}{ F. poisson } & 3570 & 3060 & & Hoshii et al $1970^{11}$ \\
\hline & 3770 & 3710 & $\mathrm{~ns}$ & Petersen et al $1976^{21}$ \\
\hline F. viande & 2980 & 2480 & s & Petersen et al $1976^{(2)}$ \\
\hline $\begin{array}{l}\text { Sous-produits } \\
\text { abattoir de volailles }\end{array}$ & 3155 & 3215 & $\mathrm{~ns}$ & Horani et Daghir $1975^{(j)}$ \\
\hline \multicolumn{5}{|l|}{$\begin{array}{l}\text { Autres matières premières } \\
\text { d'origine végétale }\end{array}$} \\
\hline \multirow[t]{2}{*}{ F. luzerne } & 1490 & 1000 & & Hoshii et al $1970^{11}$ \\
\hline & 1200 & 610 & $\mathrm{~s}$ & Petersen et al $1976^{(2)}$ \\
\hline F. d'herbe & 1497 & 1447 & $\mathrm{~ns}$ & Jonsson et Mc Nab $1983^{(7)}$ \\
\hline Pulpes de betterave & 488 & 679 & $\mathrm{~s}$ & Charalambous et Daghir $1976^{(3)}$ \\
\hline
\end{tabular}
(1) âges non précisés
(2) poules âgées de 60 semaines, poulets âgés de 3 semaines
(3) poules âgées de 85 semaines, poulets âgés de 4 semaines
(4) poules "matures", poulets âgés de 2 semaines

des nutriments, en particulier chez le jeune oiseau (Carré 1992). Les matières premières riches en protéines : tourteaux et farines animales sont également mieux valorisées par la poule, les écarts entre oiseaux pouvant atteindre $990 \mathrm{kcal} / \mathrm{kg}$. De plus, lorsque I'on dispose de plusieurs séries de données pour une même matière première, on peut constater que ces différences ne sont pas constantes : elles varient de quelques kilocalories (Hoshii et al 1970, Horani et Daghir 1975) à $990 \mathrm{kcal}$ (Petersen et al 1976) pour le tourteau de soja.

Des écarts d'amplitude variable sont également observés pour des matières premières moins classiques en alimentation aviaire : farines d'herbe, de luzerne et pulpes de betterave, seules ces dernières étant mieux valorisées par le poulet.
(5) poules âgées de 40 semaines, poulets d'âges non précisés

(6) poules âgées de 85 semaines, poulets âgés de 1 à 7 semaines

(7) poules « matures ", poulets âgés de 2 semaines
Il est classiquement admis que l'utilisation digestive de certains nutriments, en particulier les lipides, n'est pas optimale chez le jeune oiseau (voir revue de Wiseman 1984) contrairement au coq adulte. Les différences de teneur en énergie métabolisable mentionnées dans la littérature en faveur de la femelle en ponte sont-elles imputables uniquement au jeune âge du poulet du fait de l'immaturité du jeune au plan digestif ou à la poule qui utiliserait mieux l'aliment ingéré ? Pour répondre à cette question, trop peu de résultats relatifs à la comparaison coq-poule sont rapportés dans la bibliographie. En outre, les seules données disponibles sont obtenues avec une technique de bilan digestif peu adaptée à la femelle en ponte. C'est pourquoi notre premier objectif a donc été de définir et mettre en œuvre une méthode de bilan digestif adaptée à la pondeuse. 


\section{2 / Etude du bilan digestif chez la poule pondeuse}

Les méthodes conventionnelles de bilan digestif avec alimentation ad libitum et collecte totale des excreta, classiquement pratiquées chez le coq, le poulet et plus rarement chez la poule, se décomposent en deux phases. Dans la première, les oiseaux sont accoutumés à l'aliment expérimental pendant quelques jours (conditions d'équilibre) puis mis à jeun. La deuxième phase ou bilan sensus stricto, comprend une période d'alimentation contrôlée suivie d'un jeûne d'égale durée au précédent. Dans un tel schéma expérimental, les périodes de jeûne peuvent être courtes si les oiseaux sont alimentés pendant 3 à 5 jours (voir revue de Fisher et Mc Nab 1988). Ainsi, une vidange incomplète du tube digestif avant la période de bilan est compensée par le même phénomène en fin de bilan et, d'autre part, son incidence est limitée compte tenu de la grande quantité d'excreta résultant d'un niveau d'ingestion élevé. La méthode européenne de mesure de l'énergie métabolisable chez le coq et le poulet repose sur ces considérations (Bourdillon et al 1990a).

Tableau 3. Composition des aliments expérimentaux distribués en farine.

\begin{tabular}{|c|c|c|c|}
\hline Composition (\%) & Aliment A & \multicolumn{2}{|c|}{ Aliment B } \\
\hline Maïs & 25,00 & \multicolumn{2}{|c|}{68,07} \\
\hline Blé & 39,40 & & \\
\hline T. de soja & 14,00 & \multicolumn{2}{|c|}{17,90} \\
\hline Gluten de maïs & 5,44 & \multirow{2}{*}{\multicolumn{2}{|c|}{2,30}} \\
\hline Farine de viande & & & \\
\hline Farine de luzerne & 4,40 & \multirow{2}{*}{\multicolumn{2}{|c|}{1,00}} \\
\hline Px 1 & & & \\
\hline Huile de colza & 1,50 & \multicolumn{2}{|c|}{1,00} \\
\hline Carbonate de calcium & 7,80 & \multicolumn{2}{|c|}{7,60} \\
\hline Phosphate bicalcique & 1,49 & \multicolumn{2}{|c|}{1,20} \\
\hline Minéraux et vitamines & 0,90 & \multicolumn{2}{|c|}{0,90} \\
\hline DL méthionine & & \multirow{2}{*}{\multicolumn{2}{|c|}{0,03}} \\
\hline Lysine & 0,07 & & \\
\hline \multicolumn{4}{|l|}{ Caractéristiques mesurées } \\
\hline & & fabrication 1 & fabrication 2 \\
\hline Matière sèche $(\%)$ & 88,37 & 88,08 & 88,80 \\
\hline Energie brute (kcal/kg MS) & 4044 & 4082 & 4025 \\
\hline Protéines brutes (\% MS) & 20,18 & 19,51 & 19,75 \\
\hline
\end{tabular}

Tableau 4. Influence des durées de jeûne et d'alimentation sur l'énergie métabolisable apparente à bilan azoté nul : EMAN $(\mathrm{kcal} / \mathrm{kg} M S$, moyenne \pm écart-type, $n=9$ ).

\begin{tabular}{|l|c|c|c|}
\hline & \multicolumn{2}{|c|}{ Durée de jeûne } & \\
\hline Durée d'alimentation & 10 heures & 17 heures & Moyenne \\
3 jours & $2878 \pm 36^{\mathrm{a}}$ & $2938 \pm 56^{\mathrm{b}}$ & $2908^{\mathrm{A}}$ \\
7 jours & $2977 \pm 28^{\mathrm{b}}$ & $2989 \pm 46^{\mathrm{C}}$ & $2983^{\mathrm{B}}$ \\
Moyenne & $2928^{\mathrm{A}}$ & $2964^{\mathrm{B}}$ & \\
\hline
\end{tabular}

Les valeurs suivies de la même lettre ne sont pas significativement différentes à $\mathrm{P}<0,05$.

\section{1 / Période d'alimentation, période de jeûne}

\section{a / Conditions expérimentales}

Nous avons pris, pour référence, la méthode européenne qui comporte 3 jours d'alimentation et 17 heures de jeûne. Afin de limiter d'éventuelles perturbations de la ponte, nous avons réduit la durée du jeûne à 10 heures en le superposant à la période obscure du nycthémère, et augmenté la phase d'alimentation qui passe de 3 à 7 jours.

Ainsi, 4 groupes de 9 poules Warren Isabrown âgées de 32 semaines, d'un poids moyen $1800 \pm 139 \mathrm{~g}$ et placées en cage à digestibilité sont utilisées selon l'un des 4 schémas expérimentaux testés : 3 ou 7 jours d'alimentation, 10 ou 17 heures de jeûne. Ces animaux reçoivent l'aliment expérimental A (tableau 3) depuis l'âge de 20 semaines soit une adaptation de 12 semaines.

Les consommations sont mesurées individuellement, les excreta sont collectés chaque jour, stockés à $-20^{\circ} \mathrm{C}$, regroupés par oiseau puis lyophilisés. Les œufs sont comptabilisés et pesés chaque jour.

L'aliment et les féces sont analysés pour leur contenu en matière sèche, énergie brute et azote total, permettant ainsi de calculer l'énergie métabolisable apparente à bilan azoté nul $\left(\mathrm{EMA}_{\mathrm{N}}\right)$ de l'aliment.

\section{b / Résultats}

La variation des durées de jeûne et d'alimentation ne modifient pas la consommation d'aliment qui varie de 94,4 à $97,3 \mathrm{~g}$ de matière sèche (MS) par jour et par poule, pas plus que la production quotidienne d'œuf qui s'élève en moyenne à $59 \mathrm{~g}$. Les durées de jeûne et d'alimentation ont une incidence sur l'EMA $_{N}$ du régime (tableau 4). Ainsi, les valeurs sont, en moyenne, significativement plus élevées avec 17 heures de jeûne (2964 vs $2928 \mathrm{kcal} / \mathrm{kg} \mathrm{MS}$ ) et 7 jours d'alimentation (2983 vs $2908 \mathrm{kcal} / \mathrm{kg}$ MS) qu'avec $10 \mathrm{heu}-$ res de jeûne et 3 jours d'alimentation. La séquence constituée de 10 heures de jeûne et 7 jours d'alimentation conduit cependant à l'obtention d'une teneur en énergie métabolisable identique à celle obtenue avec 17 heures et 7 jours.

\section{2 / Durée d'adaptation}

\section{a / Conditions expérimentales}

Une période classique d'adaptation à l'aliment expérimental de 3 jours est comparée à une durée largement supérieure soit 7 jours. Deux lots de 12 poules Isabrown âgées de 39 semaines et d'un poids moyen de $1735 \pm$ $142 \mathrm{~g}$ sont utilisés. L'énergie métabolisable de l'aliment $\mathrm{B}$ présenté au tableau 3 est déterminée en retenant comme schéma expérimental 17 heures de jeûne et 7 jours d'alimentation après avoir accoutumé les oiseaux à l'aliment pendant 3 ou 7 jours. 
Les mesures effectuées sont les mêmes que celles décrites précédemment.

\section{b / Résultats}

Quelle que soit la durée d'adaptation des oiseaux à l'aliment expérimental : 3 ou 7 jours, les paramètres mesurés pendant le bilan digestif : consommation, variation de poids vif, poids d'œuf exporté, bilan azoté et EMA $_{N}$, ne sont pas modifiés (tableau 5).

\section{3 / Facteurs de variation de la valeur énergétique des aliments chez la poule pondeuse}

\section{1 / Influence de l'âge}

\section{a / Conditions expérimentales}

Les bilans digestifs sont pratiqués dans deux stations expérimentales : Domaine Expérimental de Sanders et Station de Recherches Avicoles de l'INRA. La conduite des bilans est identique dans les deux sites. Ils se composent de 3 jours d'adaptation, 17 heures de jeûne, 7 jours d'alimentation et 17 heures de jeûne. Les mesures et techniques mises en œuvre sont également similaires, à l'exception des fientes qui sont lyophilisées à l'INRA et déshydratées en étuve (63 heures, $80^{\circ} \mathrm{C}$ ) chez Sanders. Seul l'aliment B est utilisé (tableau 3) mais deux fabrications (1 et 2) élaborées avec des lots différents de matières premières ont été nécessaires, compte tenu de la durée des expérimentations.

Les animaux sont des poules Warren Isabrown dont l'âge varie de 16 (poulettes) à 70 semaines (poules de réforme).

Les bilans sont pratiqués sur 11 oiseaux au minimum. Les mêmes poules sont utilisées pour les bilans à 16 et 25 semaines et à 39 et 50 semaines alors que des oiseaux issus de deux troupeaux différents sont retenus pour les mesures à 28 et 70 semaines.

\section{b / Résultats (tableau 6)}

L'accroissement de l'âge des oiseaux de 16 à 70 semaines conduit à de larges variations de poids (1 $176-2030 \mathrm{~g}$ ) et de production journalière d'œuf (0-60 g/poule), les poulettes étant les plus légères et ne produisant pas, alors que les poules de réforme de 70 semaines ont le poids vif le plus élevé et conservent encore un niveau de production correct. Les consommations individuelles d'aliment varient également dans de larges proportions (65-122 g de MS/j/poule), il en est de même du bilan azoté $(0,84-1,80 \mathrm{~g} / \mathrm{j} /$ poule $)$ qui traduit à la fois la production d'cuf et le gain de protéines corporelles. L'énergie métabolisable de l'aliment la plus faible $(2996 \mathrm{kcal} / \mathrm{kg})$ est observée chez les poules âgées de 70 semaines alors que les valeurs les plus élevées sont obtenues sur des oiseaux n'ayant pas encore commencé à produire (3146 $\mathrm{kcal} / \mathrm{kg}$ à 16 semaines) ou dont la production tend à décroître $(3135 \mathrm{kcal} / \mathrm{kg}$ à 50 semaines). Les autres femelles dont l'âge se situe au pic de ponte ou juste après et donc en période de production maximale utilisent l'aliment de façon similaire (3 039-3 076 $\mathrm{kcal} / \mathrm{kg}$ ). Bien que significatives, ces variations restent faibles : $-2,6$ et $+2,3 \%$, par rapport à une moyenne qui s'élève à 3075 $\mathrm{kcal} / \mathrm{kg}$.

\section{2 / Influence du sexe}

\section{a / Conditions expérimentales}

Les énergies métabolisables des aliments A et $\mathrm{B}$ observées sur poule pondeuse sont comparées à celles obtenues sur coq adulte. Ceuxci sont nourris ad libitum selon le schéma expérimental suivant (Lessire 1990) : 3 jours d'accoutumance, 24 heures de jeûne, 2 jours d'alimentation, 24 heures de jeûne. Les excreta sont collectés en totalité les trois derniers jours. Neuf coqs sont utilisés par bilan. Ils proviennent du même croisement commercial que les poules utilisées et sont âgés de 12 et 14 mois lors de la mesure des aliments $\mathrm{A}$ et $B$ respectivement. Les analyses des aliments et des excreta sont identiques en tous points à celles pratiquées lors des essais sur femelles.

Tableau 5. Influence de la durée d'adaptation à l'aliment expérimental sur l'énergie métabolisable ( $\mathrm{kcal} / \mathrm{kg} \mathrm{MS}$ ).

\begin{tabular}{|l|c|c|}
\hline Durée d'adaptation & 3 jours & 7 jours \\
\hline Consommation $(\mathrm{g} / \mathrm{j})$ & $93 \pm 12$ & $98 \pm 9$ \\
Poids d'œuf exporté $(\mathrm{g} / \mathrm{j})$ & $60.0 \pm 4.8$ & $61.0 \pm 5.3$ \\
Bilan azoté $(\mathrm{g} / \mathrm{j})$ & $0.90 \pm 0.21$ & $0.94 \pm 0.09$ \\
EMA $_{\mathrm{N}}(\mathrm{kcal} / \mathrm{kg})$ & $3076 \pm 48$ & $3067 \pm 43$ \\
\hline
\end{tabular}

Tableau 6. Influence de l'âge de la poule sur la digestibilité de l'aliment $B$.

\begin{tabular}{|l|l|c|c|c|c|c|c|}
\hline $\begin{array}{c}\text { Age } \\
\text { (semaines) }\end{array}$ & $\begin{array}{c}\text { Lieu } \\
\text { de l'essai }\end{array}$ & $\begin{array}{c}\mathbf{N}^{\circ} \text { de fabric. } \\
\text { de l'aliment }\end{array}$ & $\begin{array}{c}\text { Poids vif } \\
(\mathbf{g})\end{array}$ & $\begin{array}{c}\text { Poids d'œuf } \\
\text { exporté (g/j) }\end{array}$ & $\begin{array}{c}\text { Consomm. } \\
\text { (g MS/j) }\end{array}$ & $\begin{array}{c}\text { Bilan azoté } \\
(\mathbf{g} / \mathbf{j})\end{array}$ & $\begin{array}{c}\text { EMA }_{\mathbf{N}} \\
(\mathbf{k c a} / \mathbf{k g} \text { MS) }\end{array}$ \\
\hline 16 & INRA & 2 & $1176 \pm 73$ & 0 & $65 \pm 6$ & $0.84 \pm 0.24$ & $3146 \pm 49 \mathrm{a}$ \\
25 & INRA & 2 & $1493 \pm 87$ & $49 \pm 5$ & $99 \pm 11$ & $1.42 \pm 0.22$ & $3039 \pm 45 \mathrm{~b}$ \\
28 & Sanders & 2 & $1725 \pm 127$ & $60 \pm 5$ & $122 \pm 11$ & $1.80 \pm 0.20$ & $3057 \pm 45 \mathrm{~b}$ \\
39 & INRA & 1 & $1735 \pm 142$ & $60 \pm 5$ & $93 \pm 12$ & $0.91 \pm 0.23$ & $3076 \pm 48 \mathrm{~b}$ \\
50 & INRA & 2 & $1816 \pm 134$ & $46 \pm 10$ & $110 \pm 13$ & $1.35 \pm 0.22$ & $3135 \pm 46 \mathrm{a}$ \\
70 & Sanders & 2 & $2030 \pm 150$ & $46 \pm 15$ & $97 \pm 19$ & $1.27 \pm 0.35$ & $2996 \pm 77 \mathrm{c}$ \\
\hline
\end{tabular}


Tableau 7. Influence du sexe de l'oiseau sur la valeur énergétique de l'aliment.

\begin{tabular}{|l|c|c|c|c|}
\hline & \multicolumn{2}{|c|}{ Aliment A } & \multicolumn{2}{c|}{ Aliment B } \\
& Poule & 52 & Coq & 60 \\
\hline Age (semaines) & 32 & $82 \pm 7$ & tableau & $107 \pm 16$ \\
Consommation (g/j) & $94.4 \pm 19.2$ & $0.07 \pm 0.05$ & 6 & $0.60 \pm 0.16$ \\
Bilan azoté (g/j) & $1.04 \pm 0.31$ & $3019 \pm 27$ & $2996-3146$ & $3162 \pm 34$ \\
EMAN (kcal/kg MS) & $2989 \pm 46$ & $3028 \pm 26$ & $3120-3259$ & $3231 \pm 34$ \\
EMA (kcal/kg MS) & $3087 \pm 57$ &
\end{tabular}

\section{b / Résultats (tableau 7)}

Les niveaux d'ingestion des deux catégories d'oiseaux sont très proches : 82 et $94,4 \mathrm{~g} / \mathrm{j}$ pour les coqs et les poules recevant l'aliment A. Il en est de même de l'aliment $B$ que les coqs consomment à raison de $107 \mathrm{~g} / \mathrm{j}$. Chez les femelles l'ingéré varie selon l'âge et les conditions expérimentales de 65 à $122 \mathrm{~g} / \mathrm{j}$ (tableau 6). Ces ingérés spontanés sont ceux classiquement admis chez des coqs ou des poules nourris ad libitum, et les variations enregistrées sont sans doute à rapprocher des conditions d'élevage pratiquées. Les bilans azotés diffèrent selon les oiseaux, ils sont largement positifs chez les femelles et proches de l'équilibre pour les coqs. Les EMAN de l'aliment A s'élèvent à 2989 et $3019 \mathrm{kcal} / \mathrm{kg}$ chez la poule et le coq respectivement. Pour le régime $\mathrm{B}$, la valeur coq (3162 kcal $/ \mathrm{kg})$ est identique aux meilleurs résultats observés chez les poulettes (3146 kcal) ou les poules âgées de 50 semaines ( $3135 \mathrm{kcal} / \mathrm{kg}$ ), les autres valeurs enregistrées chez les poules étant inférieures. Cependant, compte tenu de leur rétention azotée importante (synthèse de protéines corporelles ou exportées vers l'œuf) les EM apparentes sont en moyenne plus élevées chez la femelle.

\section{Discussion - conclusion}

Pour des conditions de mesure de l'EM des aliments optimales chez la poule, les résultats que nous obtenons en terme de durée des périodes constitutives du bilan digestif aboutissent à une méthode relativement longue : trois jours d'adaptation, 7 jours de bilan encadrés par deux jeûnes de 17 heures. A l'inverse, chez le coq, un bilan est réalisé en une semaine voire 3 jours lorsque l'on utilise une technique de gavage (Lessire 1990). Chez le poulet de chair en croissance, les bilans durent au minimum une semaine, mais l'allongement de la phase expérimentale n'aboutit pas à une réduction du coefficient de variation $(2,6 \%)$ aussi faible que celui observé chez le coq $(1,88 \%)$ (Bourdillon et al 1990b). Dans notre cas, une période expérimentale longue va de pair avec une variabilité faible $(1,65 \%)$, similaire à celle observée chez le coq. Ainsi notre schéma expérimental peut donc constituer une référence afin de mesurer en routine la valeur des aliments et matières premières destinées à la femelle en production.

En outre, l'utilisation digestive des aliments ne semble pas varier dans de grandes proportions chez cette dernière, qu'il s'agisse d'animaux en début ou en fin de ponte. En effet, l'énergie métabolisable de l'aliment équilibré que nous avons mesurée à différentes phases de la période de production de la poule n'est pas modifiée dans de grandes proportions : $\pm 2,5 \%$ autour de la valeur moyenne, même si ces différences sont significatives sans que nous puissions en fournir l'explication.

Les différences d'utilisation digestive des aliments entre sexes sont également faibles, les valeurs d'énergie métabolisable à bilan azoté nul mesurées chez le coq (EMAN) seraient cependant légèrement supérieures. Ces variations en fonction de l'âge ou du sexe de l'oiseau peuvent être attribuées à la variabilité des conditions ambiantes : saison, température, humidité, mais aussi au facteur de correction de l'EMA en EMA ${ }_{\mathrm{N}}$ qu'il conviendrait peut-être de préciser. Enfin, seul le concept d'énergie nette pourrait permettre de comparer efficacement des oiseaux à l'entretien (coq) ou en production (poule). Cependant, dans l'état actuel de nos connaissances, il conviendrait maintenant de poursuivre ces investigations par des mesures comparatives systématiques portant sur des aliments de composition plus variable, et surtout enrichis en matières grasses dont la valeur énergétique et l'absorption intestinale sont sujettes à de larges variations chez les oiseaux.

\section{Références bibliographiques}

Askbrant S., 1988. Metabolisable energy content of rapeseed meal, soya bean meal and white flowered peas determined with laying hens and adult cockerels. Br. Poult. Sci., 29, 445-455.

Bourdillon A., Carré B., Conan L., Duperray J., Huyghebaert G., Leclercq B., Lessire M., Mc Nab J., Wiseman J., 1990a. European reference method for the in vivo determination of metabolisable energy with adult cockerels : reproductibility, effect of food intake and comparison with individual laboratory methods. Br. Poult. Sci., 31, 557-565.

Bourdillon A., Carré B., Conan L., Francesch M., Huyghebaert G., Janssen W.M.M.A., Leclercq B., Lessire M., Mc Nab J., Rigoni M., Wiseman J., 
1990b. European reference method of in vivo determination of metabolisable energy in poultry : reproductibility, effect of age, comparison with predicted values. Br. Poult. Sci., 31, 567-576.

Carré B., 1992. Les polysaccharides non amylacés hydrosolubles. Conférence Avicole WPSA - SIMAVIP, Villepinte (FRA) ; 1992/03/03. In : Les contaminants et facteurs antinutritionnels dans les aliments des volailles: Vrais ou faux problèmes. Cahier $\mathrm{n}^{\circ} 8,49-58$.

Carré B., Rozo E., 1990. La prédiction de la valeur énergétique des matières premières destinées à l'aviculture. INRA Prod. Anim., 3, 163-169.

Charalambous K. V., Daghir N.J., 1976. Factors affecting the metabolizable energy values of four different poultry feedstuffs. Poult. Sci., 55, 1657-1662.

Conan L., Métayer J.P., Lessire M., Widiez J.L., 1992. Teneur en énergie métabolisable des céréales françaises pour les volailles. Synthèse d'enquêtes annuelles. INRA Prod. Anim., 5, 329-338.

Daghir N.J., Nathanaël A.S., 1974. An assessment of the nutritional value of triticale for poultry. 15th World Poultry Congress, New Orleans (USA) ; 1974/08/11-16, 612-614.

Farrel D.J., 1978. Rapid determination of metabolizable energy of foods using cockerels. Br. Poult. Sci., 19, 303-308.

Fisher C., Mc Nab J.M., 1988. Techniques for determining the metabolizable energy content of poultry feeds. In : Recent developments in Poultry Nutrition. Cole D.J.A., et Haresing W., (eds). 54-69. Butterworths, London, (GBR).

Horani F., Daghir N.J., 1975. Metabolizable energy values (M.E.) of three protein supplements as determined with chicks and laying hens. Poult. Sci., 54, 1886-1889.

Hoshii H., Setsune H., Yoshida M., 1970. Metabolizable energy of various feed ingredients estimated by quail and chicken. Jap. Poult. Sci., 7, 147-150.

Jonsson G., Mc Nab J.M., 1983. A comparison of methods for estimating the metabolizable energy of a sample of grass meal. Br. Poult. Sci., 24, 349-359.

Kussaibati R., Leclercq B., 1985. A simplified rapid method for the determination of apparent and true metabolizable energy values of poultry feed. Arch. Geflugelk., 49, 54-62.

Leclercq B., 1979. La valeur énergétique des matières premières : influence de l'âge, du sexe et du type génétique et comparaison entre espèces aviaires. In : Matières premières et alimentation des volailles. Séances de travail INRA. Nouzilly (FRA); 1979/10/18-19, 65-78.

Leclercq B., 1985. Mesure et prédiction de la valeur énergétique : Intérêt, signification, limites. Conférence Avicole WPSA-SIMAVIP, Villepinte (FRA); 1985/10/18. In : Valeur énergétique et qualité des aliments. Cahier n ${ }^{\circ}$ 1, 4-12.

Leclercq B., Lessire M., Guy G., Hallouis J.M., Conan L., 1989. Utilisation de la graine de colza en aviculture. Revue bibliographique et résultats de deux essais. INRA Prod. Anim., 2, 129-136.

Lessire M., 1990. Effect of feeding technique, ad libitum dry or wet force feeding, on the metabolizable energy values of raw materials for poultry. $\mathrm{Br}$. Poult. Sci., 31, 785-793.

Lessire M., Leclercq B., Conan L., 1988. Variabilité de la valeur énergétique de la graine de soja traitée chez les volailles, INRA Prod. Anim., 1, 265-270.

Lilburn M.S., Jensen, L.S., 1984. Evaluation of corn fermentation solubles as a feed ingredient for laying hens. Poult. Sci., 63, 542-547.

Lodhi G.N., Renner R., Clandinin D.R., 1969. Studies on the metabolizable energy of rapeseed meal for growing chickens and laying hens. Poult. Sci., $48,964-970$

Parson C.M., Potter L.M., Bliss B.A., 1982. True metabolizable energy corrected to nitrogen equilibrium. Poult. Sci., 61, 2241-2246.

Pesti G.M., 1994. Description d'un modèle de formulation permettant un profit maximum. Conférence avicole WPSA-SIMAVIP, Villepinte (FRA), 1994/2/15 ; In Nutrition et Alimentation Avicoles; vers une démarche intégrée. Cahier n 9, 25-46.

Petersen C.F., Meyer G.B., Sauter E.A., 1976. Comparison of metabolizable energy of feed ingredients for chicks and hens. Poult. Sci., 55, 1163-1165.

Sibbald I.R., 1976a. A bioassay for true metabolizable energy in feedingstuffs. Poult. Sci., 55, 303308.

Sibbald I.R., 1976b. The true metabolizable energy values of several feedingstuffs measured with roostes, laying hens, turkeys and broiler hens. Poult. Sci., 55, 1459-1463.

Wiseman J., 1984. Assessment of the digestible and metabolizable energy of fats for non-ruminants. In : Fats in animal nutrition. Wiseman J., ed. ; 277-297. Butterworths, London, (GBR).

\begin{abstract}
Metabolisable energy value of diets in laying hens.

Adult cockerels are classically used for metabolisable energy evaluation of poultry diets. The obtained data are retained by feed manufacturers in order to formulate diets for growing birds and laying hens. But it has been demonstrated that cockerel is not always the right model for young birds. In the present review we show that it may be the same for laying hens even if the results obtained are difficult to compare due to

variations in the methodologies used for the ME determinations.

In the first trial we define the fasting and feeding periods of the bioassay in the second one we determined the effect of the age of the hen (from 16 to 70 weeks) on the ME value of an experimental diet and compared the obtained data to those determined on adult cockerels.

LESSIRE M., REVOL N., RUDEAUX F., HALLOUIS J.-M., 1995. Valeur énergétique des aliments chez la poule pondeuse. INRA Prod. Anim., 8 (3), 189-195.
\end{abstract}

